\title{
Carrera de Tecnología Superior en Ciencias Militares y su vinculación con comunidades rurales: Caso Seguridad y Cuidado del Medio Ambiente

\author{
Higher Technology Program in Military Sciences and its relationship with \\ rural communities: Case, Safety and Environmental Care
}

\section{ROSANNA PAULINA BARBA CEVALLOS (D), MAGALY MARGARITA NARVÁEZ RÍOS*(D), FLOR EMPERATRIZ GARCÉS MANCERO DD, JEFFERSON JOFFRE BARROS SANTAMARIA}

Universidad de las Fuerzas Armadas ESPE,

Av. General Rumiñahui y Ambato, 170501, Sangolquí, Ecuador

*mmnarvaez5@espe.edu.ec

\section{RESUMEN}

El presente manuscrito evidencia las intervenciones para desarrollar habilidades y capacidades en la conservación del medio ambiente a través de la reflexión en torno a una educación ambiental, permitiendo así la participación activa y mejoramiento en la protección ambiental, y a la vez facilitar la inclusión de los estudiantes de la Carrera de Tecnología Superior en Ciencias Militares en su Modalidad Dual, perteneciente a la Escuela de Formación de Soldados "Vencedores del Cenepa", en la solución de problemas sociales que están afectando a las comunidades rurales beneficiarias: Salcedo, Quero y Píllaro a través del proyecto de vinculación con la sociedad. Este proyecto tiene como objetivo generar espacios comunitarios de participación que contribuyan a garantizar una mejor calidad de vida, fortaleciendo la relación civil-militar en los ámbitos de intervención e interrelación entre las instituciones participantes. Mediante la aplicación de instrumentos de investigación empleamos el procedimiento de la técnica de la encuesta en las áreas de intervención de las comunidades rurales, en donde el efecto esperado es elevar el nivel de la cultura de seguridad y cuidado ambiental. Analizados los resultados, se determinó que la población beneficiaria maneja la aplicación de principios, medidas y procedimientos de protocolos de seguridad, concerniente a gestión de riesgos y 
un medio ambiente sano a las presentes y futuras generaciones con poco conocimiento, resaltando la problemática del cambio climático, los desastres naturales, la pérdida de biodiversidad y la erosión de la diversidad biocultural.

Palabras Claves: Educación ambiental, medio ambiente, reforestación, seguridad, comunidades, desastres antrópicos.

\section{ABSTRACT}

This research shows the interventions to develop skills and capacities in the conservation of the environment through reflection on environmental education to allow active participation and improvement in environmental protection, and at the same time, to facilitate the inclusion of students from the Higher Technology Program in Military Sciences in its Dual Modality, which belongs to the Soldiers Training School "Vencedores del Cenepa" in solving social problems that are affecting to the beneficiary rural communities of Salcedo, Quero, and Pillaro through the social project with the community, which aims to generate community spaces for participation by contributing to guarantee a better quality of life by strengthening the civil-military interrelationship in the areas of intervention and interrelation between the participating institutions. Through the application of research instruments, we use the procedure of the survey technique for intervening areas in the rural communities, where the expected effect is to raise the level of the culture of safety and environmental care. After analyzing the results, it was determined that the beneficiary population manages the application of principles, measures, and procedures of security protocols, concerning with risk management and a healthy environment for present and future generations with little knowledge by highlighting the problem of climate change, natural disasters, loss of biodiversity, and the erosion of biocultural diversity.

Keywords: Environmental education, environment, reforestation, security, communities, anthropic disasters.

\section{INTRODUCCIÓN}

Dentro de los objetivos estratégicos de la Defensa Nacional (Ministerio de Defensa Nacional, 2018) se encuentra establecido el objetivo $N^{\circ}{ }^{\circ}$ 2, que señala: El apoyo a las instituciones en la protección de la población en sus derechos, libertades ante graves situaciones de conmoción interna y de situaciones de desastre. En el Ecuador, por su posición geográfica, se encuentra sometido a diversas amenazas naturales; las amenazas que mayor impacto socio-económico han causado son las inundaciones, los eventos sísmicos, los volcánicos y los movimientos de masas o deslizamientos. Los habitantes de zonas rurales son afectados por ciertas dificultades que limitan su desarrollo integral, el aislamiento geográfico y la poca aplicación de programas comunitarios de acuerdo a las necesidades de la población. Varias son las causas que provocan impedimentos para obtener una mejor calidad de vida, por lo tanto, es importante fortalecer la identidad de los habitantes, reforzando sus valores y el desarrollo de sus destrezas; en función de aquello en este estudio se abordó la importancia y el potencial que tiene la situación actual en las áreas de intervención, el mismo que está siendo ejecutado por la Escuela de Formación "Vencedores del Cenepa" considerando a 
los pobladores pertenecientes a las zonas rurales-marginales en los cantones de las provincias de: Chimborazo y Tungurahua, los mismos que desconocen sobre los efectos negativos de desastres de origen natural o antrópico, gestión de riesgos, educación ambiental, valores e identidad nacional, y que no cuentan con la capacitación e inducción necesaria para mitigar la degradación ambiental.

La iniciativa para realizar este análisis surge al valorar que la cultura no es un eje más del desarrollo, sino el eje de referencia, por lo que la idea central es poner énfasis en aquellos aspectos que limitan el avance del desarrollo comunitario rural, sobre todo considerando que la comunidad rural es el único escenario posible donde sus habitantes pueden realizar un trabajo consciente, integrado, participativo, planificado, organizado y coordinado que les permita mejorar su calidad de vida.

Para el efecto, la ESPE-ESFORSE dispone de Talento Humano en: instructores militares, docentes civiles, estudiantes de segundo año militar y tutores académicos que facilitarán la ejecución del proyecto, es importante mencionar que el método de enseñanza-aprendizaje de los aspirantes es de modalidad dual, lo que implica que los estudiantes permanezcan una parte del tiempo en el aula donde es la ciencia técnica de lo aprendido y por otra parte estarán involucrados directamente en lo práctico, que se realizará por toda la carrera militar, basándose en la alternancia de los tiempos y los espacios educativos de los alumnos de formación profesional, dada por aplicarse en la Escuela de Formación de Soldados "Vencedores del Cenepa" ESFORSE.

\section{DESARROLLO}

Durante la última década, hechos acontecidos como el terremoto del año 2016, la reactivación de volcanes Cotopaxi, Tungurahua, Sangay dentro del territorio continental y el volcán La Cumbre en la Isla Fernandina en el Archipiélago de Galápagos, pérdida de espacios verdes en diferentes regiones, inundaciones, entre otros fenómenos naturales de menor magnitud evidenciaron la vulnerabilidad de la población ecuatoriana y la dificultad para enfrentar y mitigar con efectividad los efectos provocados por los eventos señalados previamente.

La temática referente a seguridad ha observado un incremento en cuanto a la importancia atribuida por parte de la ciudadanía y por entes gubernamentales. Si bien es cierto que el tópico aludido es sumamente amplio, se hace mención específicamente a lo denominado como cultura de seguridad o cultura de prevención. De acuerdo con (Díaz, 2017), quien efectúa un estudio sobre el aspecto cultural de la prevención en Perú, menciona que ha sido establecida una política pública que se orienta al manejo y mitigación de riesgos que garanticen un estado de protección e invulnerabilidad para los ciudadanos; no obstante, especifica que es imperativo constituir estamentos que se dirijan a instancias de previsión de peligros y amenazas sociales a través de la educación. En efecto (Sura, 2020) alude que una cultura de seguridad se define como un sistema de conocimientos y predisposición para ejecutar actividades dirigidas a: previsión ante el suceso o no de un fenómeno natural o humano, convenio y cooperación para fomentar el mantenimiento de un estado de salud adecuado por parte de organizaciones familiares, educativas y comunitarias. Como 
se puede apreciar, la cultura de seguridad de un país no se enfoca únicamente en las acciones destinadas a paliar los efectos de un desastre natural o fenómenos provocados por el ser humano, sino que se extiende a programar y organizar un sistema de prevención de riesgos.

Dentro de este contexto, la gestión de riesgos hace parte fundamental del macro-concepto referente a la cultura de seguridad. De acuerdo con el (Programa de las Naciones Unidas, PNUD Chile, 2012) la gestión de riesgos se rige como el procedimiento caracterizado por una serie de estamentos, tácticas y acciones dirigidas a impedir, disminuir y contrarrestar los impactos ocasionados por un evento o fenómeno natural - humano, su campo de acción de la gestión de riesgos enfatiza sobre el desarrollo de destrezas y acciones que brinden servicio y ayuda ante un desastre de determinado tipo y a la capacitación de estrategias de preparación ante un acontecimiento específico, de este modo, en Ecuador la gestión de riesgos se ha convertido en un punto esencial dentro de la planificación de los organismos encargados de este eje. Según (Bernabé, 2015) durante la última década, la gestión de riesgos en Ecuador pasó de ser un campo de soluciones al momento de gestarse un evento natural a un sistema de acciones orientadas a la previsión, control y mitigación de los consecuentes originados por causa de un fenómeno de la naturaleza o humano. Como es evidente, la adopción de una cultura de seguridad o cultura de prevención en territorio ecuatoriano ha sido un proceso paulatino, el cual ha expuesto cambios mayoritariamente positivos al concentrar esfuerzos en prever los efectos que pueden provocar los distintos tipos de acontecimientos.
La implementación de las denominadas "buenas prácticas agrícolas" (Gutiérrez-Guzmán et al., 2012) incluyendo la agricultura ecológica y la agricultura de conservación, entre otras, son capaces de recuperar ciertos niveles de biodiversidad y de servicios ecosistémicos distintos a la producción agrícola respecto a las prácticas convencionales. La transformación de los agroecosistemas en bosques y matorrales por el abandono de cultivos y pastizales que son sujeto de la regeneración natural o en plantaciones forestales por las reforestaciones de tierras agrícolas (Benayas \& Fraile, s.f.).

La identidad nacional atraviesa por momentos de cambios por el acontecimiento de una diversidad de eventos suscitados al interior de la geografía ecuatoriana. Si bien es cierto que la transmisión cultural permanece latente en tiempos vigentes, es indiscutible que el arribo de una heterogeneidad de componentes pertenecientes a factores identitarios extranjeros ejerce una influencia considerable sobre la cultura de los ecuatorianos. Según (Arias, 2009) la identidad nacional se encuentra sujeta a procesos externos al espacio geográfico de una sociedad, por ejemplo, la extensión de la globalización a nivel mundial ha erigido un sin número de transformaciones en distintos niveles: políticos, manejo de política económica y aspecto cultural. Al hacer alusión al territorio ecuatoriano, la enseñanza de determinados aspectos culturales se halla supeditada en cierta magnitud al sistema educativo. De acuerdo con (De la Cruz, 2008) a través de la enseñanza de textos, el objetivo del sistema educativo ecuatoriano no se enfatiza en transferir un conjunto de pautas y normas enraizadas en un sentimiento nacionalista, sino que acerca a los sujetos a establecer la consecución de un estado subjetivo, esclarecido sobre información comprobada 
respecto a la existencia de etnias heterogéneas, costumbres, valores y tradiciones del país; así como informar sobre el conjunto de derechos y responsabilidades de un individuo y asociación familiar. Tal y como se aprecia, la enseñanza en establecimiento educativos sobre determinados aspectos de la identidad nacional es importante. No obstante, la familia es el ente primario que se encarga de la transmisión de dichos aspectos culturales.

\section{METODOLOGÍA}

El enfoque aplicado en la investigación es cualitativo con un estudio descriptivo, la modalidad del proceso comprende investigación bibliográfica de campo y una propuesta de intervención que aporta significativamente a la comunidad educativa; con el sustento de la bibliografía citada permitió fortalecer el conocimiento para que el proyecto tenga viabilidad técnica y se encuentre garantizada en medida de las actividades planteadas donde se fundamentan en la formación teórico-metodológica y técnico-instrumental de los estudiantes de la Carrera de Tecnología Superior en Ciencias Militares, que se sustentan en la doctrina y asignaturas aprobadas.

Para facilitar la conducción de talleres y trabajo comunitario, se realizó una encuesta a la población de las comunidades de Quero, Píllaro y Salcedo para poder realizar una orientación adecuada y que se apliquen diferentes técnicas participativas sobre los temas de Gestión de Riesgos, la Educación Ambiental e Identidad Nacional.

\section{DIAGNÓSTICO}

Como primera actividad al analizar los objetivos planteados cuyo propósito es dar a conocer la situación de los comuneros en el cantón de Quero, Píllaro y Salcedo, se ve la necesidad de desarrollar el plan de trabajo para las comunidades vulnerables de cada cantón, es por ello que se plantean las acciones y recursos que se establecen en la siguiente tabla.

\section{Tabla 1}

Plan de Trabajo con las comunidades vulnerables

\begin{tabular}{|c|c|c|c|}
\hline $\mathbf{N}^{\circ}$ & Objetivos & Acciones & Recursos \\
\hline 1 & $\begin{array}{c}\text { Reforzar el conocimiento } \\
\text { de la población en los } \\
\text { temas relacionados con } \\
\text { fenómenos naturales y } \\
\text { antrópicos. }\end{array}$ & $\begin{array}{l}\text { Realizar charlas de manera paulatina en } \\
\text { los temas propuestos y relacionados } \\
\text { para prevenir riesgos y mejorar el } \\
\text { accionar de la población }\end{array}$ & $\begin{array}{c}\text { Personal } \\
\text { capacitado } \\
\text { lugar adecuado y } \\
\text { materiales } \\
\text { necesarios } \\
\end{array}$ \\
\hline 2 & $\begin{array}{l}\text { Dar a conocer las } \\
\text { distintas zonas seguras } \\
\text { que existen en cada } \\
\text { cantón. }\end{array}$ & $\begin{array}{l}\text { Recorrer junto a la población los } \\
\text { diferentes sitios seguros en donde se } \\
\text { pueda salvaguardar la vida de las } \\
\text { personas y, mediante una dinámica, } \\
\text { realizar una demostración de lo que } \\
\text { podría suceder. }\end{array}$ & $\begin{array}{l}\text { Población, } \\
\text { personal } \\
\text { capacitado, } \\
\text { personal de } \\
\text { apoyo. Sitios } \\
\text { amplios y seguros }\end{array}$ \\
\hline 3 & $\begin{array}{l}\text { Informar a la población } \\
\text { qué tipo de problemas } \\
\text { ambientales podemos } \\
\text { tener y cómo podemos } \\
\text { ayudar a disminuir su } \\
\text { impacto }\end{array}$ & $\begin{array}{l}\text { Ejecutar exposiciones acerca de los } \\
\text { problemas ambientales y emplear junto a } \\
\text { los ciudadanos programas de apoyo } \\
\text { para disminuir los impactos negativos al } \\
\text { medio ambiente. }\end{array}$ & $\begin{array}{c}\text { Personal } \\
\text { capacitado, } \\
\text { población, } \\
\text { lugar adecuado y } \\
\text { materiales } \\
\text { necesarios }\end{array}$ \\
\hline 4 & $\begin{array}{l}\text { Instruir sobre valores, } \\
\text { derechos y deberes a la } \\
\text { población, lo cual es } \\
\text { necesario para mantener } \\
\text { una mejor convivencia } \\
\text { social. }\end{array}$ & $\begin{array}{l}\text { Trabajar junto con la comunidad en } \\
\text { programas de desarrollo e inclusión } \\
\text { basados en valores para mantener una } \\
\text { buena actitud con sus compañeros o } \\
\text { vecinos, respetando la pluriculturalidad. }\end{array}$ & $\begin{array}{c}\text { Personal } \\
\text { capacitado, } \\
\text { población, } \\
\text { lugar adecuado }\end{array}$ \\
\hline
\end{tabular}

Nota. Acciones y recursos para los objetivos planteados para la situación de los comuneros en el cantón de Quero, Píllaro y Salcedo. 
Con el plan de trabajo descrito se utilizan los objetivos específicos como medio para que los comuneros y los directivos de los cantones de Quero, Píllaro y Salcedo conozcan la situación y los criterios que se quiere abordar dentro de cada comunidad, y a partir de eso saber cuáles son los puntos fuertes y cuáles son las áreas de intervención donde es preciso realizar la ayuda en temas de la gestión de riesgos, la reforestación como parte del cuidado del medio ambiente y el rescate de valores; a partir de ese cotejo se pueden implantar las mejores acciones de mejora dentro de las comunidades.

\section{RESULTADOS}

Como primer punto se identificó la población objetivo de forma directa, tanto hombres como mujeres, donde se puede observar una totalidad de 67.235 habitantes directos en los 3 cantones, de los cuales se cuenta con un grupo de 31.878 hombres y un grupo de 35.357 mujeres. Igualmente, tenemos una totalidad de 173,809 habitantes indirectos basados en los 3 cantones (Tabla 2).

\section{Tabla 2}

Identificación de la población

Nota. Población objetivo de forma directa hombres y mujeres y totalidad de habitantes indirectos.
Se trabajó con los estudiantes de segundo año militar la instrucción por personal altamente capacitado del ECU-911, permitiendo aplicar los conocimientos para emplear en situaciones de emergencia. La finalidad del taller fue multiplicar la información recibida por los capacitadores hacia los beneficiarios de los sectores más vulnerables en áreas rurales de las provincias de Cotopaxi y Tungurahua como: Salcedo, Quero y Píllaro impartiendo charlas sobre cuidado y protección ambiental; de igual forma se impartieron temas del valor a los símbolos patrios en las áreas de intervención, que serán aplicadas por los pobladores de manera técnica.

Las salidas de intervención para la reforestación se realizó en el cantón Salcedo, donde el alcalde precisó el área de intervención en el sector de Salache Angamarca, donde se identificaron sectores desprotegidos de vegetación, con el trabajo conjunto de 30 habitantes y el personal de estudiantes se procedió a la siembra, haciendo uso de palas, picos y machetes, Promoción 2018-2020, llevando plantas de invernaderos donde realizaron el cavado de hoyos para la plantación de especies ornamentales dadas en la misma provincia para la regeneración y sembrío de vegetación nativa para su medio ambiente, por último se hizo una limpieza de $360^{\circ}$ alrededor de la planta.

En el cantón Quero se realizó la reforestación de las laderas del sector y su limpieza, sembrando plantas nativas como: cholanas, acacias, cepillo blanco, cepillo rojo, fuma maqui, jacaranda, entre otras variedades más de la zona, realizando los hoyos distanciados para que los árboles puedan expandirse y tomar mejor forma en su crecimiento. Se empleó el recurso humano necesario y se obtuvo la colaboración de algunos habitantes del sector, dando así la seguridad y concretando que se ha realizado la actividad en el área de intervención. 
Figura 1

Plantación y regadío


Nota. Cantón Quero se realizó la reforestación de las laderas del sector y su limpieza, sembrando plantas nativas.

Los efectos en cascada sobre las especies nativas pueden resultar de la reducción simultánea de la cantidad total de vegetación nativa y la conectividad del paisaje. Varios estudios han sugerido que la pérdida de conectividad es particularmente severa por debajo del umbral del $30 \%$ de la cubierta vegetal nativa, lo que lleva a un aumento de las pérdidas de especies que dependen de esta vegetación. Por lo tanto, "la regla del 30 \%" no se aplica a todas las especies ni a todos los ecosistemas (Parker y Mac Nally, 2002 ; Lindenmayer et al. , 2005), (Fischer \& Lindenmayer, 2007).

En este sentido, la protección del medio ambiente constituye una necesidad actual para toda la humanidad, y debe ser considerada como un objetivo educativo que se lleva a cabo en las instituciones universitarias. Es por ello que la interacción del hombre con la naturaleza es una condición básica e indispensable para la existencia y desarrollo de la sociedad. Sin embargo, dependiendo cómo sea este nexo, planificado o arbitrario, racional o irracional, consciente o espontáneo, así será también el futuro del medio natural en que habita el hombre y, por consiguiente, el de la sociedad.
En el cantón Píllaro se realizó la actividad de gimnasia de alteres brindando homenaje a Rumiñahui en el Mirador de Huaynacuri, lugar natal del mítico héroe indígena, en cuyo honor se ha edificado un monumento de granito al que acuden sus habitantes año tras año a celebrar las fiestas del Inti Raymi. Demostraron la destreza, preparación física y energía de cada aspirante al realizar una actividad con entusiasmo y emoción, la misma que es un atractivo a la vista de los espectadores (habitantes del cantón), se logró rescatar los valores cívicos y militares en la población, lo que será una motivación para fortalecer el compromiso tanto de la comunidad como de la institución.

Figura 2

Oración de la Gimnasia

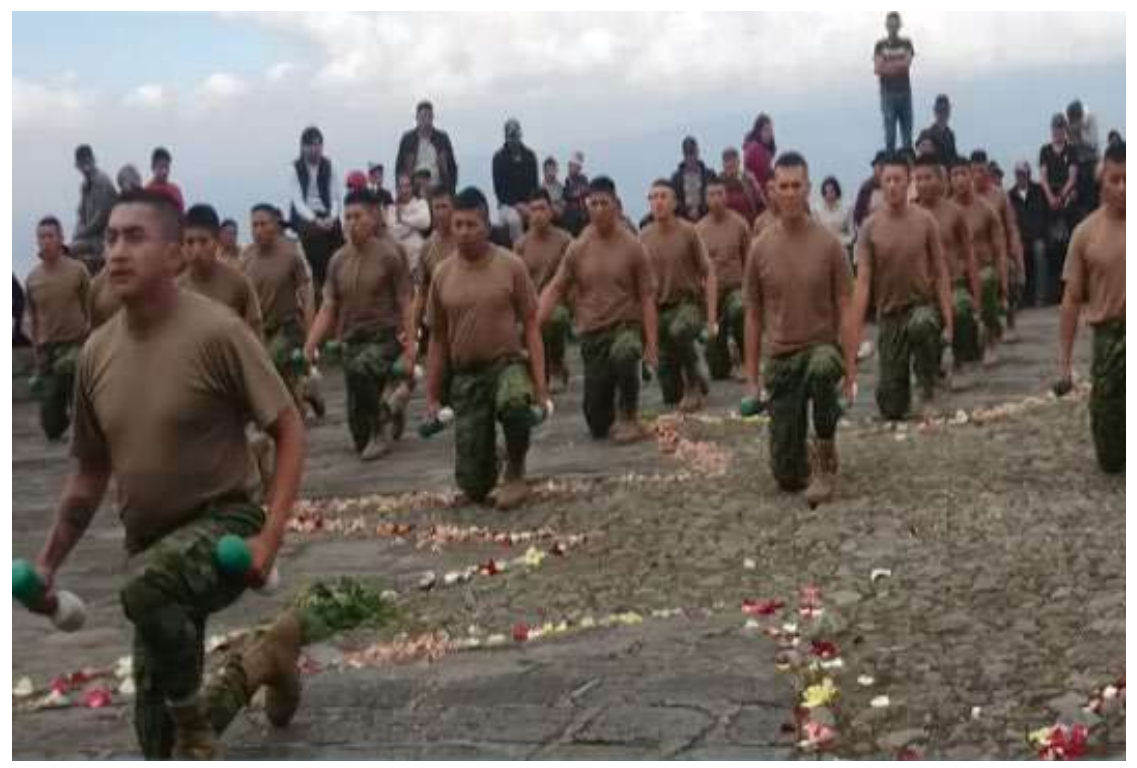

Nota. Cantón Píllaro se realizó la actividad de gimnasia de alteres brindando homenaje a Rumiñahui en el Mirador de Huaynacuri. 
Los estudiantes de la Carrera de Tecnología Superior en Ciencias Militares en la entidad formadora ESFORSE hacen la oración símbolo de cada gimnasia, una vez que han terminado se da un grito de aliento y motivación. Los involucrados han tenido un rol muy importante en este programa, teniendo en cuenta que la Escuela de Formación de Soldados "Vencedores del Cenepa" (ESFORSE) y sus instructores militares pudieron aumentar su participación en los programas de vinculación ya realizados. Los docentes y las autoridades de la ESFORSE lograron colaborar en la ejecución y desarrollo del proyecto, de tal manera que los estudiantes participantes mejoren sus competencias y conocimientos a través de su intervención.

Se realizó una encuesta a través de un cuestionario en el cual podemos evidenciar que la aplicación del sistema de actividades y acciones dirigidas por los estudiantes universitarios de la Carrera de Tecnología Superior en Ciencias Militares a las comunidades es positiva, cumpliendo así con el propósito de la construcción de una educación ambiental y de un desarrollo sostenible en las zonas intervenidas. Los resultados se dieron a conocer mediante gráficos demostrando profesionalismo, responsabilidad y comprometimiento con la solución de los problemas de su entorno y de la sociedad. Se demostrarán sus competencias a través del desempeño y la actuación ciudadana digna de los más altos valores morales.

\section{Figura 3}

Encuesta a beneficiarios

¿̇Ha recibido Ud. capacitación sobre "GESTIÓN DE RIESGOS" por parte de alguna institución de educación superior?

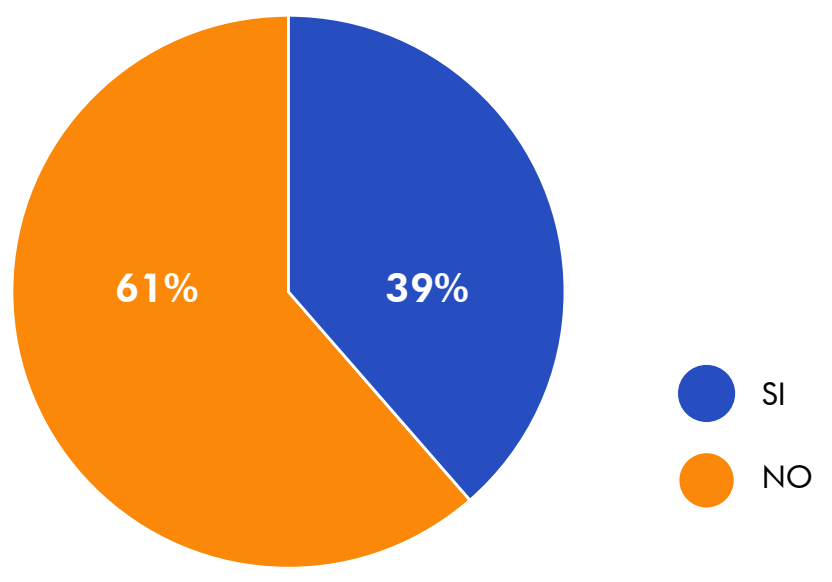

$\dot{2}$ Considera que se debe continuar con el trabajo de vinculación en su parroquia?

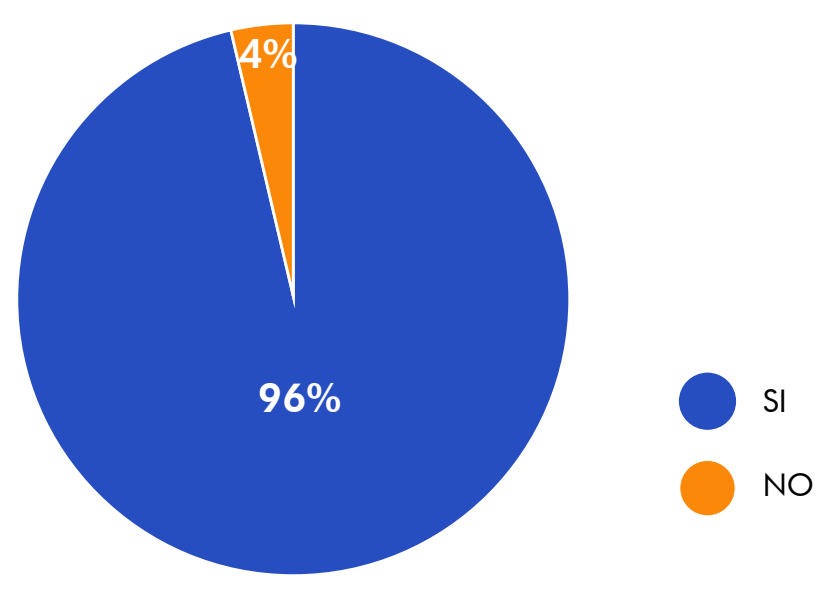


¿Observa que el trabajo de vinculación de la ESFORSE deja un aporte significativo al conocimiento de los pobladores de la parroquia?

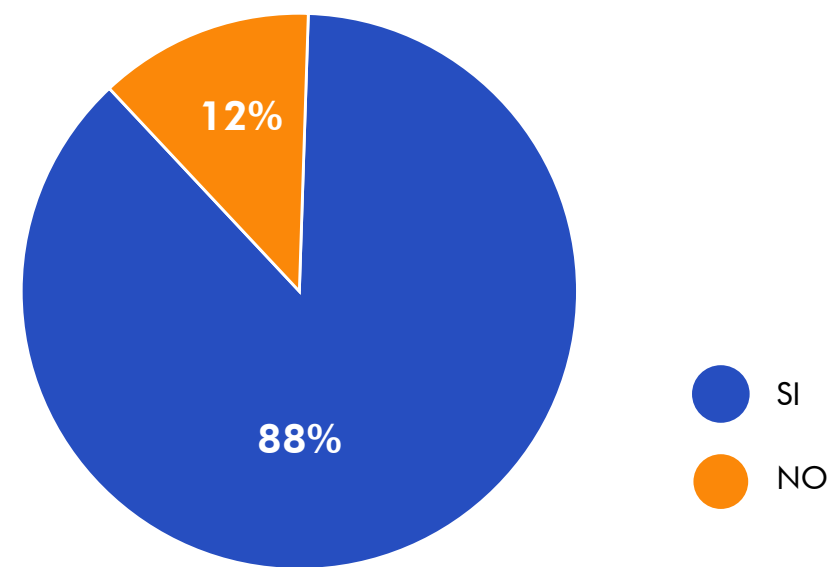

¿Cree que el aporte de los señores aspirantes a soldados tecnólogos de la ESFORSE, aportan con conocimientos en beneficio de su comunidad?

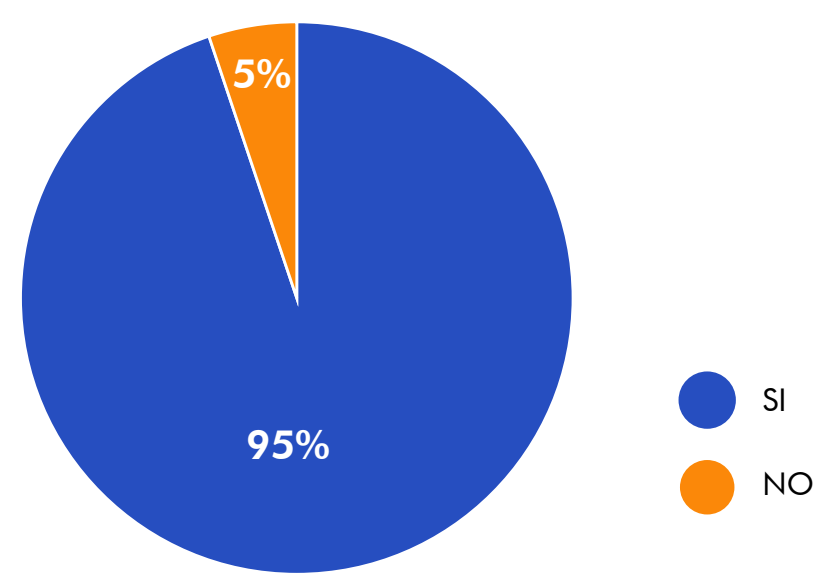

Nota. Encuesta a través de un cuestionario en el cual se puede evidenciar que la aplicación del sistema de actividades y acciones dirigidas por los estudiantes universitarios de la Carrera de Tecnología Superior en Ciencias Militares a las comunidades es positiva.
Los GAD cantonales y parroquiales junto con la comunidad de beneficiarios directos e indirectos mostraron desactualización y falta de conocimiento sobre la actuación eficiente ante los fenómenos naturales antrópicos y gestión de riesgo, por ende, pudieron recibir el beneficio de este programa que fue desarrollado con la participación de la comunidad, cabe recalcar que cada uno de los involucrados tuvo un recurso o mandato al que se rigió para poder cumplir de manera correcta este proyecto.

\section{CONCLUSIONES}

La elaboración de programas de capacitación y educación ambiental para concientizar a la población de comunidades rurales como mecanismo de incorporación progresiva a la problemática ambiental fue posible gracias al empleo de métodos dinámicos para el tratamiento y cuidado del medio ambiente.

La consecución de los objetivos permitirá realizar un desarrollo rural sostenible, centrado en el uso consciente de los recursos naturales en medida de sus posibilidades, fortaleciendo el conocimiento, comunicación, organización, participación, solidaridad y el compromiso de los comuneros. Quedó plasmada la iniciativa de los aspirantes para que sigan brindando el apoyo a las comunidades rurales en el área del cuidado del medio ambiente, compromiso que asume la institución militar como parte de sus objetivos previstos, así mismo, se reflejaron los valores que se fomentan dentro del instituto por medio de la gimnasia de alteres. 
Se fortaleció la responsabilidad e identidad social de la comunidad, recalcando que los procedimientos desarrollados no provocarán una afectación directa al medio ambiente de la zona de intervención del proyecto.

Se logró la sostenibilidad social destinada para la comunidad de los cantones de Tungurahua y Cotopaxi sin distinción de clase social o de género, motivando a todos los habitantes a mejorar el conocimiento sobre la aplicación de principios, medidas y procedimientos de protocolos de seguridad, gestión de riesgos y seguridad ambiental.

\section{AGRADECIMIENTO}

Extendemos un agradecimiento a las autoridades de la escuela y GADs Municipales por su apertura y colaboración con el proyecto, el cual se lo hizo en beneficio de la comunidad, partiendo del contexto social y de acuerdo a sus necesidades. Esperamos que nuestro servicio de capacitación en el área de seguridad integral haya sido un aporte para generar espacios de áreas reforestadas que mitiguen la degradación ambiental manteniendo y conservando sus áreas naturales.

\section{REFERENCIAS BIBLIOGRÁFICAS}

Arias, L. (2009). La identidad nacional en tiempos de globalización. Revista Electrónica Educare, 13(2), 7-16.

Bernabé, M. B. (2015). Gestión de Riesgo en el Ecuador. Sangolquí: Editorial ESPE.

De la Cruz, P. (2008). Enseñando identidad nacional en el Ecuador. MINIUS, 113-134
Díaz, J. (2017). Promoción de una cultura de prevención de accidentes. Horizonte de la Ciencia, 7(13), 83-88.

Ecuadorencifras.gob.ec. (15 de ENERO de 2020). Obtenido de https://www.ecuadorencifras.gob.ec/documentos/webinec/Bibliotecas/Fasciculos_Censales/Fasc_Cantonales/ Tungurahua/Fasciculo_Quero.pdf

Jaramillo, R. (2014). Ciudadanía, Identidad Nacional y EstadoNación. Revista Lasallista de Investigación, 11 (2), 168-180.

Programa de las Naciones Unidas, PNUD Chile. (2012). Conceptos Generales sobre Gestión del Riesgo de Desastres y Contexto del País. Chile: Gráfica Troya.

Sura, P. W. (2020). ARL Sura. Obtenido de https://www.arlsura. com/index.php/component/content/article/66-centro-dedocumentacion-anterior/prevencion-de-riesgos-/1470-iquees-cultura-de-prevencion.

Benayas, J. M. R., \& Fraile, A. M. (n.d.). Diagnóstico y directrices para la restauración de agroecosistemas.

Fischer, J., \& Lindenmayer, D. B. (2007). Landscape modification and habitat fragmentation: a synthesis. Global Ecology and Biogeograph, 16(3), 265-280. https://doi.org/https://doi. org/10.1111/j.1466-8238.2007.00287.x

Gutiérrez-Guzmán, N., Serra, J. A., \& Dussan-Sarria, S. (2012). Priorización de factores críticos para implantar buenas prácticas agrícolas en pequeños productores * Cuadernos de Desarrollo Rural, 9(69), 221-237.

Ministerio de Defensa Nacional. (2018). Política de la Defensa Nacional del Ecuador "Libro Blanco" (S. Guerra (ed.); Vol. 66). 


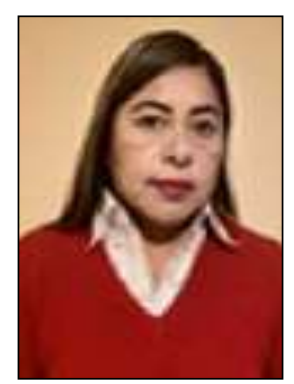

\section{ROSANNA PAULINA BARBA CEVALLOS}

Doctora en Gerencia Educativa, Máster en Docencia Universitaria y Administración Educativa, Docente de la Unidad Académica Especial ESFORSE, Coordinadora de Vinculación con la Sociedad de la Carrera de Tecnología Superior en Ciencias Militares ESPE. Ha escrito 5 artículos indexados, ponente y participante en congresos nacionales e internacionales. Integrante de comités evaluadores en proyectos de investigación.

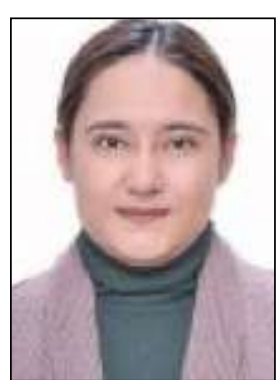

\section{MAGALY MARGARITA NARVÁEZ RÍOS}

Ingeniera en Sistemas Informáticos y Computación, Master en Investigación en Ingeniería de Sistemas y de la Computación, Docente de la Unidad Académica Especial ESFORSE. Ha escrito 13 artículos indexados en Scopus y Latinlndex, ha sido ponente en congresos nacionales e internacionales. Forma parte de comités científicos de revistas Scopus JCR Q3.

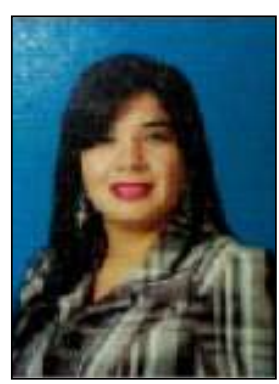

\section{FLOR EMPERATRIZ} GARCÉS MANCERO

Ingeniera en Banca y Finanzas, Máster en Diseño y Formulación de Proyectos Sociales y Productivos. Está realizando su Tesis Doctoral en Gestión Pública y Gobernabilidad, ha laborado en instituciones públicas en el área Financiera y Educación (Universidad de las Fuerzas Armadas ESPE) y ha impartido formación en las Escuelas Militares ESMA y ESFORSE.

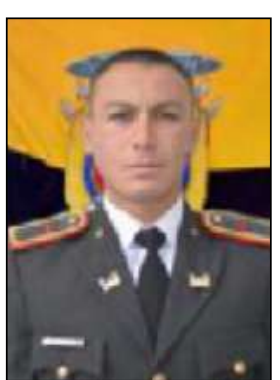

JEFFERSON JOFFRE BARROS SANTAMARIA

Inicia su carrera militar en 2018 ingresando a la Escuela de Formación de Soldados del Ejército "Vencedores del Cenepa". Se gradúa como Soldado del Ejército Ecuatoriano en agosto de 2020 y actualmente ejerce sus funciones en la provincia de Zamora - Ecuador. Ha realizado varios cursos de Certificación en Aviación Inicial y temas semejantes. 